\title{
Direct preparation of green and renewable aerogel materials from crude bagasse
}

\author{
Mingjie Chen $\cdot$ Xueqin Zhang • \\ Aiping Zhang $\cdot$ Chuanfu Liu $\cdot$ Runcang Sun
}

Received: 30 January 2015/Accepted: 3 November 2015/Published online: 22 January 2016

(C) The Author(s) 2016. This article is published with open access at Springerlink.com

\begin{abstract}
Lignocellulosic biomass aerogels with relatively high Brunauer-Emmett-Teller (BET) surface area and pore volume were prepared directly from bagasse solutions in the present study. Bagasse was dissolved in $\mathrm{DMSO} / \mathrm{LiCl}$, treated with cyclic freezingthawing processes, and regenerated with water. The resulted bagasse hydrogels were solvent-exchanged to t-butanol and subjected to freeze-drying to obtain the lignocellulosic aerogels. The structure of the aerogels was studied with FTIR, nitrogen absorption, SEM, and XRD. The results showed that the aerogels had sheetlike skeletons interconnected with one another to form three-dimensional networks. The highest BET surface area and total pore volume of the aerogels were
\end{abstract}

Electronic supplementary material The online version of this article (doi:10.1007/s10570-015-0814-9) contains supplementary material, which is available to authorized users.

M. Chen · X. Zhang $\cdot$ C. Liu $(\bowtie) \cdot$ R. Sun

State Key Laboratory of Pulp and Paper Engineering,

South China University of Technology,

Guangzhou 510640, China

e-mail: chfliu@gmail.com

\section{A. Zhang}

Institute of New Energy and New Material, Guangdong

Key Laboratory for Innovative Development and Utilization of Forest Plant Germplasm, South China

Agricultural University, Guangzhou 510642, China

R. Sun

Beijing Key Laboratory of Lignocellulosic Chemistry, Beijing Forestry University, Beijing 100083, China
$185 \mathrm{~m}^{2} / \mathrm{g}$ and $0.46 \mathrm{~cm}^{3} / \mathrm{g}$, respectively. By adjusting the concentration of bagasse solution in $\mathrm{DMSO} / \mathrm{LiCl}$, the structure of the aerogels could be controlled to some extent.

Keywords Lignocellulosic biomass - Biobased materials · Green materials · Aerogels · Freeze-drying

\section{Introduction}

Aerogels are solid materials prepared by replacing the liquid solvent in a gel by gas without substantially collapsing the gel solid network (Tan et al. 2001). The first aerogel could be dated back to 1931 (Kistler 1931), which was prepared with supercritical drying. Since then, a great deal of aerogel materials have been developed, all of which can be divided into three types: inorganic, inorganic-organic and organic aerogels (Tan et al. 2001). Because of the extremely low densities, large micropore volumes, and high inner surface area, aerogels have met extensive application in catalyst supports, films, separation, chemical analysis, sensing, absorbents, energy technologies, and energy absorption (Hüsing and Schubert 1998).

The most common aerogels are inorganic aerogels (for example, silica and alumina aerogels). Organic aerogels represent an interesting alternative to the inorganic aerogels (Guo et al. 2012) and can be subsequently converted to carbon aerogels by pyrol ysis, which are the only electron conductive aerogels. 
Resorcinol/formaldehyde (RF) and melamine/formaldehyde (MF) aerogels prepared by sol-gel chemistry represent the most widely studied organic aerogels (Pierre and Pajonk 2002). However, because of the harmfulness and high cost of the precursors involved in these syntheses, the large-scale production of these organic aerogels has never been allowed for industrial application, which drives researchers to look for facile, economic, and environmentally friendly raw materials to prepare organic aerogels. Nowadays, aerogels prepared from biomass materials have attracted significant attentions (Wu et al. 2013) due to their renewability, low cost, abundance, nontoxicity to humans, etc.

Aerocellulose is the most attractive aerogel materials from biomass due to the following considerations: (1) cellulose is one of the most abundant renewable polymer (Klemm et al. 2005), (2) excellent performance of aerocellulose (Cai et al. 2008) and subsequent transformation into carbon aerogel with desirable properties (Hao et al. 2014). In general, the preparation of aerogels from cellulose could be divided into three steps (Liebner et al. 2008; Sescousse et al. 2011): dissolution of lignocellulose, coagulation in non-solvents, and transformation of wet gels to aerogels (drying). Among these steps, the dissolution is prerequisite. Therefore, resea rches on developing renewable aerogel materials were focused merely on cellulose, and none on lignocellulosic biomass which were believed to be insoluble in conventional solvents due to the complicated structure of lignocellulosic biomass.

Lignocellulosic biomass in the form of plant (such as wood, grasses and agricultural residues) is a natural composite of cellulose, lignin, hemicelluloses and extractives, and represents the most abundant renewable resources on earth. Sustainable green composites derived from biobased, biodegradable, and renewable biomass as alternatives to traditional petroleum-based composite materials have attracted more and more attentions (Nagarajan et al. 2013). It would be more attracted to develop engineering materials directly from lignocellulosic biomass than from cellulose since the isolation of cellulose always involves in hardzous chemicals and environmental pollution. Highly compatible wood thermoplastic composites were prepared from lignocellulosic materials upon chemical modification in ionic liquid (IL) 1-butyl-3-methylimidazolium chloride (Xie et al. 2009). Biomass composite fibers were successfully prepared directly from lignocelluloses (southern yellow pine and bagasse) in IL 1-ethyl-3-methylimidazolium acetate with a dry-jet wet spinning process (Sun et al. 2011). Lignocellulosic biomass film was also prepared directly from bagasse solution in our laboratory (Chen et al. 2014). These achievements of biomass materials directly from lignocellulose indicate that the direct conversion of lignocelluloses in novel solvents could be an important method to prepare biomaterials.

Lignocellulosic biomass aerogels were firstly prepared by dissolving spruce wood in IL 1-butyl-3methylimidazolium chloride followed by coagulation in aqueous ethanol with supercritical $\mathrm{CO}_{2}$ drying (Aaltonen and Jauhiainen 2009). The spruce wood aerogels were consisted of nanofibrillar biomaterial network with Brunauer-Emmett-Teller (BET) surface area as high as $122 \mathrm{~m}^{2} / \mathrm{g}$, and Barrett-Joyner-Halenda (BJH) adsorption average pore diameter ranging from 25 to $30 \mathrm{~nm}$. However, there was limit information on the detailed structure of the wood aerogels. The preparation of lignocellulosic biomass aerogels were also achieved from wood solutions in 1-allyl-3methylimidazolium chloride with cyclic freezingthawing process and supercritical $\mathrm{CO}_{2}$ drying $(\mathrm{Li}$ et al. 2011; Lu et al. 2012). Aerogels with high BET surface area are desirable in many industrial applications such as functional carriers (Sehaqui et al. 2011). However, the reported lignocellulosic biomass aerogels suffered from low BET surface area, among which the highest one was only $122 \mathrm{~m}^{2} / \mathrm{g}$ (Aaltonen and Jauhiainen 2009; Li et al. 2011; Lu et al. 2012).

More recently, another novel solvent system $\mathrm{DMSO} / \mathrm{LiCl}$ was developed for the complete dissolution of milled wood (Wang et al. 2009), providing the possibility to prepare lignocellulosic aerogels with increased properties. In the present study, lignocellulosic biomass aerogels with high BET surface area were prepared from bagasse solutions in $\mathrm{DMSO} / \mathrm{LiCl}$ with cyclic freezing-thawing processes and t-butanol freeze-drying techniques. The physico-chemical properties of the bagasse aerogels were characterized by FTIR, nitrogen absorption, SEM, and XRD.

\section{Experimental}

\section{Materials}

Bagasse was obtained from a local factory (Jiangmen, China). It was dewaxed with toluene-ethanol $(2: 1, \mathrm{v} / \mathrm{v})$ 
for $12 \mathrm{~h}$ and ball-milled with a planetary ball mill (PM100, Retsch, Germany) for $4 \mathrm{~h}$. DMSO, $\mathrm{LiCl}$ and t-butanol were of analytical-reagent grade from Aladdin Reagent Co. (Shanghai, China). Water used in the experiments was ultrapure water from a purification system (Direct-Q3, Millipore, USA). Ethanol was analytical-reagent and purchased from Guangdong Guanghua Sci-Tech Co., Ltd (Guangzhou, China).

\section{Preparation of aerogels}

The lignocellulosic aerogels were prepared from crude bagasse according to the following procedure. Ballmilled bagasse $(0.5 \mathrm{~g})$ was suspended in $9.5 \mathrm{~g} \mathrm{DMSO} /$ $\mathrm{LiCl}$ solvent system with $6 \%$ weight ratio of $\mathrm{LiCl}$ at $110{ }^{\circ} \mathrm{C}$ with agitation in oil bath for $5 \mathrm{~h}$ under $\mathrm{N}_{2}$ atmosphere to guarantee the complete dissolution of bagasse. Polarized microscopy (OLYMPUS BX-51) was applied to ensure the dissolution of bagasse. The bagasse solution was cast onto a glass mold and subjected to four cyclic freezing-thawing treatments. In each cycle, the bagasse solution was frozen at $-35{ }^{\circ} \mathrm{C}$ for $5 \mathrm{~h}$ and thawed at room temperature (approximately $30{ }^{\circ} \mathrm{C}$ ) for $5 \mathrm{~h}$. The frozen-thawed bagasse solution was immersed in water as the coagulation bath to obtain bagasse hydrogel. The water in bagasse hydrogel was solvent-exchanged several times to ethanol, and then to t-butanol. The gel containing t-butanol was freezed at $-35{ }^{\circ} \mathrm{C}$ for $12 \mathrm{~h}$ and subjected to freeze-drying (room temperature, 14.0 Pa) to obtain bagasse aerogel.

\section{Characterization}

Porosity, density and shrinkage of bagasse aerogel were determined according to a previous study (Heath and Thielemans 2010) using an analytical balance (0.1 mg accuracy), a digital calliper $(0.001 \mathrm{~mm}$ accuracy) and a measuring cylinder $(0.1 \mathrm{~mL}$ accuracy). The volume of $10 \mathrm{~g}$ bagasse solution $\left(\mathrm{V}_{\mathrm{s}}\right)$ was $8.90 \mathrm{~mL}$. Bagasse was dissolved DMSO/LiCl, coagulated in water, dried in $105{ }^{\circ} \mathrm{C}$ oven for $12 \mathrm{~h}$ to obtain samples for measuring the skeletal density of aerogel $\left(\rho_{\mathrm{s}}\right)$. The $\rho_{\mathrm{s}}$ was determined according to ISO 1183-1:2004. The $\rho_{\mathrm{s}}$ was $1.51 \mathrm{~g} / \mathrm{cm}^{3}$, and independent on bagasse concentration. The porosity, density and shrinkage of bagasse aerogel were calculated according to the following Eqs. (1-4): $\rho_{\mathrm{s}}=\mathrm{m}_{(\text {bagasse })} / \mathrm{V}_{\mathrm{s}}$

$\rho_{(\text {aerogel })}=\mathrm{m}_{(\text {aerogel })} / \mathrm{V}_{(\text {aerogel })}$

$\mathrm{P}=\left(1-\rho_{(\text {aerogel })} / \rho_{\mathrm{s}}\right) \times 100$

$\mathrm{S}=\left(1-\mathrm{V}_{(\text {sample })} / \mathrm{V}_{\mathrm{s}}\right) \times 100$

where $\rho_{\mathrm{s}}$ is the theoretical density of aerogel without any shrinkage, $\mathrm{m}_{\text {(bagasse) }}$ is the mass of bagasse dissolved in $\mathrm{DMSO} / \mathrm{LiCl}, \mathrm{V}_{\mathrm{s}}$ is the volume of bagasse solution in $\mathrm{DMSO} / \mathrm{LiCl}, \rho_{\text {(aerogel) }}$ is the measured density of the aerogel samples, $\mathrm{m}_{\text {(aerogel) }}$ is the mass of the measured aerogel sample, $\mathrm{V}_{\text {(aerogel) }}$ is the volume of the measured aerogel sample, $\mathrm{P}$ is the porosity of the measured aerogel, and $\mathrm{S}$ is the shrinkage of the aerogel sample.

BET-surface area and BJH-pore dimensions of the aerogels were determined from nitrogen adsorptiondesorption isotherms on an ASAP2020 Physisorption Analyzer from Micromeritics Instrument Corporation (USA) (Groen et al. 2003). SEM was conducted on a LEO 1530 VP (LEO, Germany) with an accelerating voltage of $10 \mathrm{kV}$. FTIR spectra were recorded on an FTIR spectrophotometer (Tensor 27, Bruker, Germany) using a $\mathrm{KBr}$ disc containing $1 \%$ finely ground samples. Thirty-two scans were taken for each sample in the region $4000-400 \mathrm{~cm}^{-1}$ at a resolution of $4 \mathrm{~cm}^{-1}$ in the transmittance mode. The XRD analysis was conducted on a D8 Advance X-ray diffractometer (Bruker, Germany) equipped with Ni-filtered $\mathrm{Cu} \mathrm{K} \alpha_{1}$ radiation $(\lambda=0.154 \mathrm{~nm})$ at room temperature. The scattering angle range was $5-40^{\circ}$ with $8 \%$ min scanning speed and a $2 \theta$ step interval of $0.02^{\circ}$.

\section{Results and discussion}

Formation of aerogel from bagasse

The dissolution of bagasse was performed accor ding to a previous study (Wang et al. 2009) with minor modification. Bagasse was decrystallised by ball-milled treatment, and subsequently dissolved in $\mathrm{DMSO} / \mathrm{LiCl}$. The bagasse/DMSO/LiCl mixture (Fig. 1a) was keep stirred at $110{ }^{\circ} \mathrm{C}$ until a semitransparent brown solution was achieved (Fig. 1b). To further confirmed the totally dissolution of bagasse, 

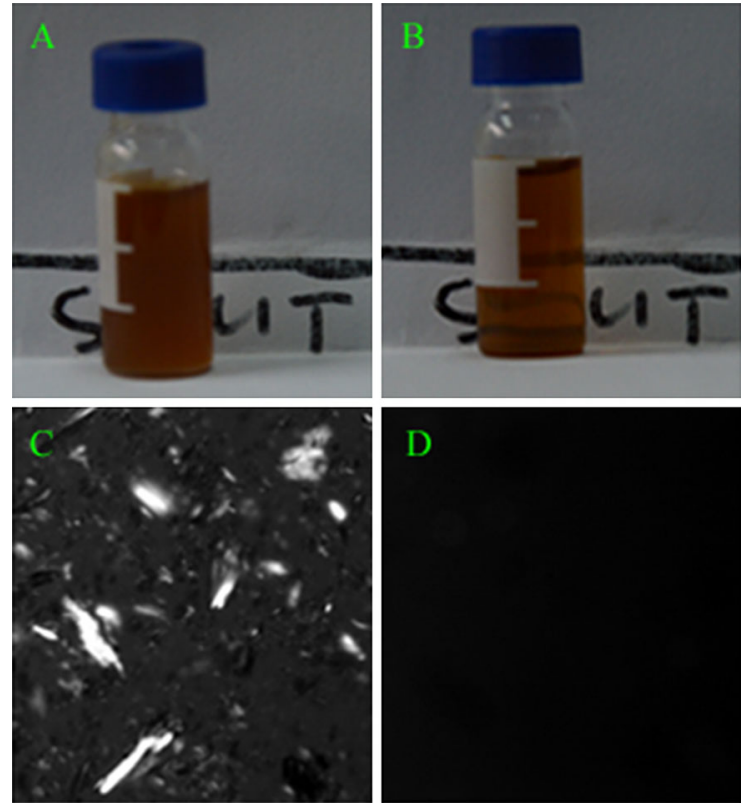

Fig. 1 Dissolution of bagasse in $\mathrm{DMSO} / \mathrm{LiCl}$. a Photo of bagasse dispersed in DMSO; $\mathbf{b}$ photo of bagasse dissolved in $\mathrm{DMSO} / \mathrm{LiCl}$, after $5 \mathrm{~h}$ heated at $110{ }^{\circ} \mathrm{C}$; c polarized microscope picture of bagasse dispersed in $\mathrm{DMSO} / \mathrm{LiCl}$ (from a), $\times 100$; d polarized microscope picture of dissolved bagasse (from $\mathbf{b}$ ), $\times 100$

polarized microscope study was performed (Fig. 1c, d). There were bright eyeshots under polarized microscope analyses due to the present of crystalline cellulose in bagasse. Upon the complete dissolution of bagasse, the field of polarized microscope turned dark black totally.

The dissolution of bagasse in $\mathrm{DMSO} / \mathrm{LiCl}$ resulted in a low viscosity solution, and no gelation was observed as the concentration of bagasse in DMSO/ $\mathrm{LiCl}$ was lower than $7 \%$. As a result, it was difficult to form bagasse hydrogel upon coagulating bagasse solution in water with the concentration of bagasse lower than $7 \%$, subsequently led to the failure of transformation of bagasse into aerogel. Freezingthawing treatment was applied to prepare bagasse aerogel according to the previous research ( $\mathrm{Li}$ et al. 2011). Even though, bagasse hydrogel could not be formed at bagasse concentration $1 \%$. Bagasse hydrogels were obtained at the concentration of $3 \%$ with freezing-thawing treatment. The bagasse hydrogels were subjected to solvent-exchange and then to freezedrying to obtain the bagasse aerogels (Fig. 2). The following discussion will base on the aerogels prepared by the freezing-thawing pretreatment processes.

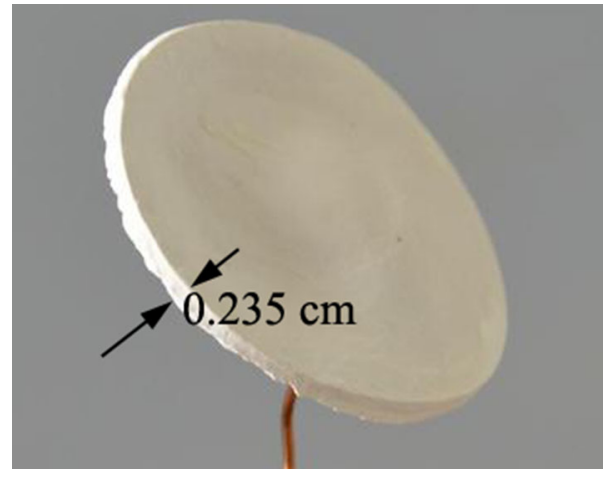

Fig. 2 Example of a bagasse aerogel sample from $5 \%$ bagasse in $\mathrm{DMSO} / \mathrm{LiCl}$ solution

Porosity, bulk density and shrinkage of bagasse aerogels

The porosities of bagasse aerogels was in the range from 84.4 to $94.2 \%$. The bulk shrinkage of hydrogels was about $40 \%$, and had no clear relationship to bagasse concentration. No further shrinkage took place upon solvent-exchange treatment, except that of the one with bagasse concentration $3 \%$ (Table 1). The total shrinkage of aerogel samples ranged from 42.7 to $59.2 \%$. Though there were no evident relation between shrinkage and concentration, high concentration tended to small shrinkage (Fig. 3). There were linear relationship between bulk density and concentration (Fig. 3). The density of bagasse aerogel ranged from 0.088 to $0.236 \mathrm{~g} / \mathrm{cm}^{3}$, which could be compared with those of cellulose aerogel $\left(0.06-0.3 \mathrm{~g} / \mathrm{cm}^{3}\right)$ prepared from cellulose/ $\mathrm{NaOH}$ aqueous solutions (Gavillon and Budtova 2008). It should be interesting to fabricating materials directly from bagasse with properties similar to cellulose. The measured density of aerogels was significantly larger than the theoretical density due to the shrinkage of the aerogels. It is suggested that limiting the shrinkage is the key to obtain aerogels with low density.

Nitrogen adsorption analysis

In order to characterize the pore structure of the bagasse aerogels, the Nitrogen adsorption-desorption isotherms were measured at $77 \mathrm{~K}$ and are illustrated in Fig. 4. According to IUPAC classification (Sing 1985), the absorption-desorption isotherms obtained were of type IV and hysteresis type H3 loop. Type IV 
Table 1 Porosity, bulk density and shrinkage of bagasse aerogels

\begin{tabular}{llllll}
\hline Concentration $(\mathrm{w} / \mathrm{w})(\%)$ & Porosity $(\%)$ & $\mathrm{S}_{\mathrm{h}}{ }^{\mathrm{a}}(\%)$ & $\mathrm{S}_{\mathrm{e}}{ }^{\mathrm{b}}(\%)$ & $\mathrm{S}_{\mathrm{b}}{ }^{\mathrm{c}}(\%)$ & $\mathrm{S}_{\mathrm{a}}{ }^{\mathrm{d}}(\%)$ \\
\hline 1 & - & - & - & - & - \\
3 & 94.2 & 40.9 & 48.1 & 48.1 & 57.8 \\
5 & 90.9 & 39.7 & 39.7 & 39.7 & 59.2 \\
7 & 88.5 & 40.9 & 40.9 & 40.9 & 52.6 \\
10 & 84.4 & 40.9 & 40.9 & 40.9 & 42.7 \\
\hline
\end{tabular}

${ }^{a}$ The shrinkage of bagasse hydrogels

b The shrinkage of ethanol contained gels

c The shrinkage of t-butanol contained gels

$\mathrm{d}$ The shrinkage of aerogels

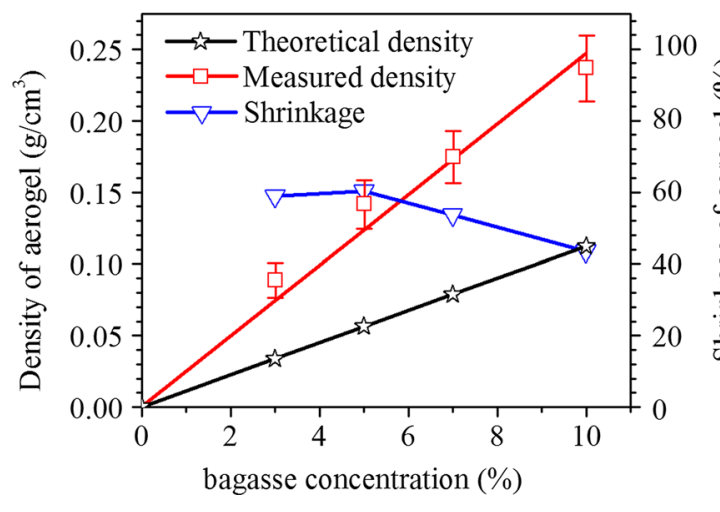

Fig. 3 Plotting of density and shrinkage to concentration

involves monolayer-multilayer adsorption on mesoporous adsorbents in a non-porous form with strong adsorbate-adsorbent interaction, and the type $\mathrm{H} 3$ loop indicates the aggregates of plate-like particles giving rise to slit-shaped pores. The rapidly increased amount of adsorbed nitrogen near the relative pressure $\left(\mathrm{P} / \mathrm{P}_{0}\right)$ of 1.0 indicated large mesopore volume. These results were similar to those of cellulose aerogels, which were prepared by gelation of cellulose from aqueous alkali hydroxide/urea solution followed by supercritical $\mathrm{CO}_{2}$ drying (Cai et al. 2008).

The specific surface area of the bagasse aerogels was determined from the adsorption isotherms at relative pressures below 0.3 using BET analysis (Table 2). The results showed that the bagasse aerogels had high specific surface area with a maximum of $185 \mathrm{~m}^{2} / \mathrm{g}$, substantially higher than those of lignocellulosic aerogels $\left(2-7.5 \mathrm{~m}^{2} / \mathrm{g}\right)$ prepared from wood solution in IL by cyclic freezing-thawing process

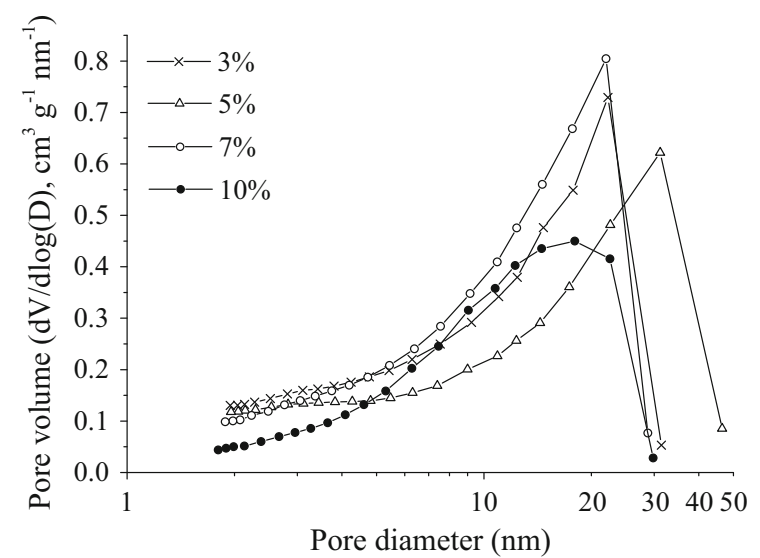

Fig. 4 Nitrogen adsorption-desorption isotherms of the bagasse aerogels

followed by supercritical $\mathrm{CO}_{2}$ drying ( $\mathrm{Li}$ et al. 2011; Lu et al. 2012), which was the most effective drying method to prepare homogeneous cellulose aerogels (Hoepfner et al. 2008). The BET surface area of the prepared bagasse aerogels was also higher than that of the wood aerogel $\left(80.7 \mathrm{~m}^{2} / \mathrm{g}\right)$ prepared from hardwood in IL 1-allyl-3-methylimidazolium chloride with 5 cyclic freezing-thawing treatment ( $\mathrm{Lu}$ et al. 2012) and that of wood aerogel $\left(122 \mathrm{~m}^{2} / \mathrm{g}\right)$ prepared from spruce wood solutions in IL 1-butyl-3-methylimidazolium chloride with supercritical carbon dioxide drying (Aaltonen and Jauhiainen 2009), and nearly comparable to those of cellulose aerogels (190$213 \mathrm{~m}^{2} / \mathrm{g}$ ) prepared from $\mathrm{DMSO} / \mathrm{LiCl}$ solutions via solvent exchange drying (Wang et al. 2012). The high BET surface area of the aerogels suggested that $\mathrm{DMSO} / \mathrm{LiCl}$ is an ideal solvent system to prepare aerogels from crude bagasse. 
Table 2 BET surface area and pore structural parameters of the bagasse aerogels

\begin{tabular}{llllll}
\hline Concentration $(\mathrm{w} / \mathrm{w})(\%)$ & $\mathrm{S}_{\mathrm{BET}}\left(\mathrm{m}^{2} / \mathrm{g}\right)$ & $\mathrm{d}_{\mathrm{A}}^{\mathrm{a}}(\mathrm{nm})$ & $\mathrm{V}_{\text {total }}^{\mathrm{b}}\left(\mathrm{cm}^{3} / \mathrm{g}\right)$ & $\mathrm{V}_{\text {meso }}{ }^{\mathrm{c}}\left(\mathrm{cm}^{3} / \mathrm{g}\right)$ & $\begin{array}{l}\text { \% Mesopore } \\
\text { volume }\end{array}$ \\
\hline 1 & - & - & - & - & - \\
3 & 185 & 9.35 & 0.44 & 0.39 & 89 \\
5 & 156 & 11.40 & 0.44 & 0.37 & 84 \\
7 & 185 & 9.50 & 0.46 & 0.42 & 91 \\
10 & 119 & 9.46 & 0.32 & 0.29 & 90 \\
\hline
\end{tabular}

${ }^{\mathrm{a}} \mathrm{BJH}$ average pore diameter determined with the nitrogen adsorption branch

b Total pore volume determined at $\mathrm{P} / \mathrm{P}_{0}$ of 0.99

c Mesopore volume determined at $\mathrm{P} / \mathrm{P}_{0}$ of 0.95

d The percentage of $V_{\text {meso }}$ to $V_{\text {total }}$ determined according to the reference (Heath et al. 2013)

There was not a clear correlation between the BET surface area and bagasse concentration in $\mathrm{DMSO} / \mathrm{LiCl}$ in the range of 3-7\%. The similar results were reported in the preparation of cellulose nanowhisker aerogels (Heath and Thielemans 2010). However, the increase of bagasse concentration from 7 to $10 \%$ led to a decrease in BET surface area from 185 to $119 \mathrm{~m}^{2} /$ g. Because BET surface area describes the area accessible to gas adsorption and is dependent on the extent of aggregation of bagasse (Heath et al. 2013), the noticeable decrease of BET surface area suggested the significant bagasse aggregation at bagasse concentration $10 \%$.

The pore properties of the bagasse aerogels including the mesopore volume (from $\mathrm{N}_{2}$ adsorption measurements), total pore volume and the percentage of mesopore are also shown in Table 2. The results showed that the bagasse aerogels with the total pore volume as high as $0.46 \mathrm{~cm}^{3} / \mathrm{g}$ could be obtained under the selected conditions. Similar to BET surface area, the total pore volume significantly decreased at bagasse concentration $10 \%$, which was probably due to bagasse aggregation at high concentration. The percentage of mesopore volume varied from 84 to $91 \%$, indicating large amount of mesopore present in the bagasse aerogels. However, the relationship between percentage of mesopore volume and bagasse concentration in all range studied was not clear.

To further study the structure of the aerogels, BJH method was applied to analyze the nitrogen absorption isotherm data. The $\mathrm{BJH}$ adsorption average pore diameter was in the range of $9.35-11.40 \mathrm{~nm}$ and didn't significantly change with the increased bagasse concentration. As shown in Fig. 5, the bagasse aerogels possessed wide mesopore size distributions, mainly in the range of $2-30 \mathrm{~nm}$. In comparison to cellulose aerogels prepared from $\mathrm{DMSO} / \mathrm{LiCl}$ solution (Wang et al. 2012), the bagasse aerogels displayed a narrow mesopore distribution and small average pore diameter. The difference was probably attributed to the freezing-thawing treatment applied in the preparation of bagasse aerogels.

Morphology of the aerogels studied with SEM

Considering the low sensitivity of nitrogen sorption experiments to large pore sizes (Sehaqui et al. 2011), the porosity of bagasse aerogels were further studied with SEM (Figs. 6, 7). Asymmetrical porosity of the aerogels was clearly observed from the SEM images. The morphology of the bottom side faces contacted by glass mold showed more dense structure (Fig. 6) with nanopores (Fig. 7). The images obtained from cross section indicated that the aerogels were composed of sheet-like skeletons interconnected with one another to form three-dimensional networks with large interstitial spaces. This finding was consistent with the results from nitrogen adsorption data analysis described above. The upside in the contact with the air of aerogels prepared at lower bagasse concentration had large pore structure consisting of sheet-like skeletons (Fig. 6). Increasing bagasse concentration to $7 \%$, the upside of aerogels showed the hierarchical morphology structure with micro/nano-scale pores. Further increase of bagasse concentration to $10 \%$ resulted in a coarse and dense upside of aerogel with pore diameter at nano-scale (Fig. 6), suggesting the extensive aggregation of bagasse, which was in 
Fig. 5 BJH pore size distribution of the bagasse aerogels
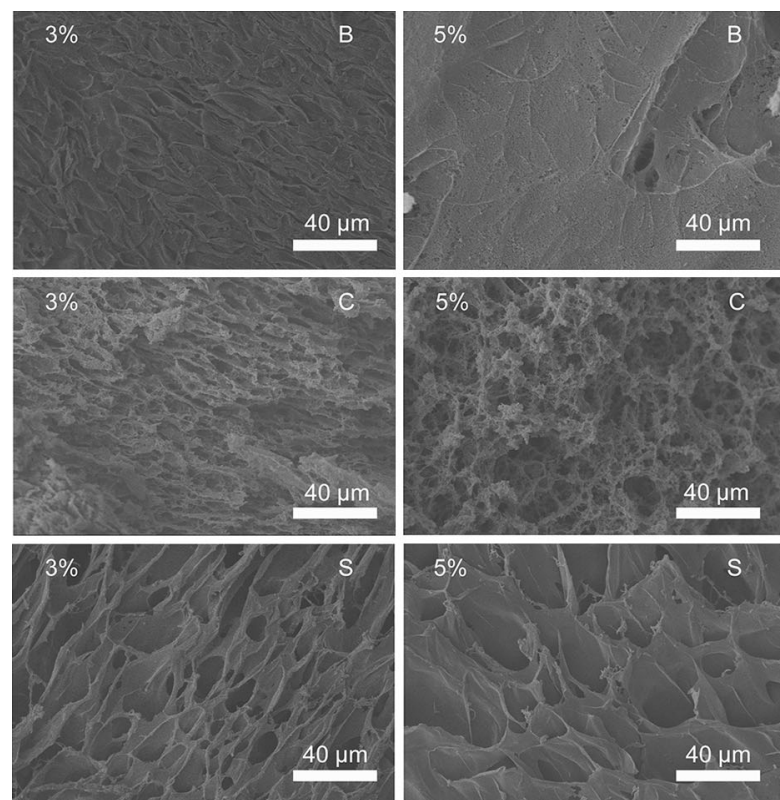
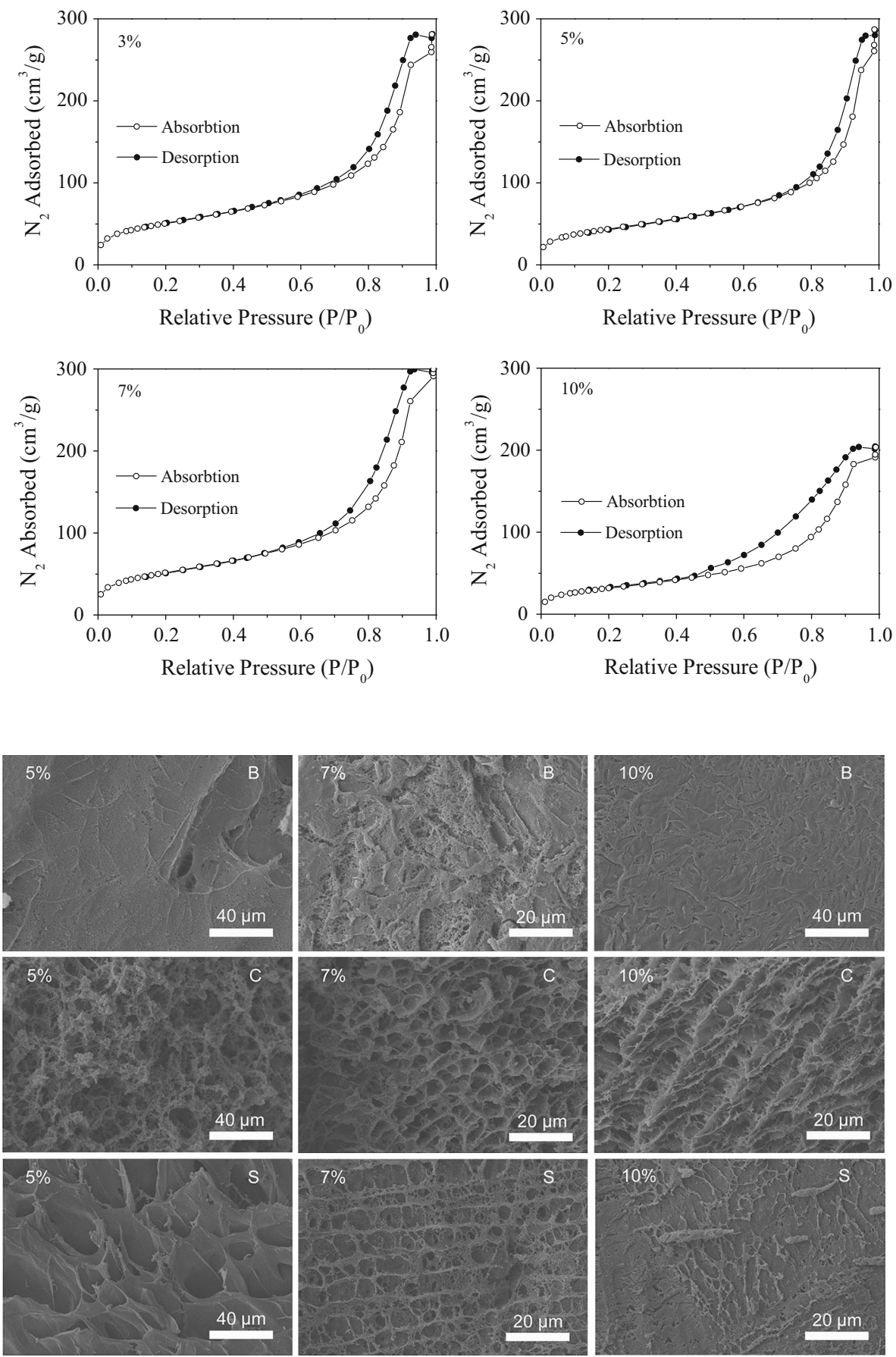

Fig. 6 SEM pictures of the bagasse aerogels at low magnification. $B, C$, and $S$ refer to bottom side (the face contacted by mold), cross section, and upside surface of the aerogels, respectively

agreement with the results from BET surface area analysis. Figure 7 were SEM images from bottom side and upside of bagasse aerogels at high magnification.
The results showed that there were no significant difference between the upside and bottom at nanoscale in the scope of this research. 

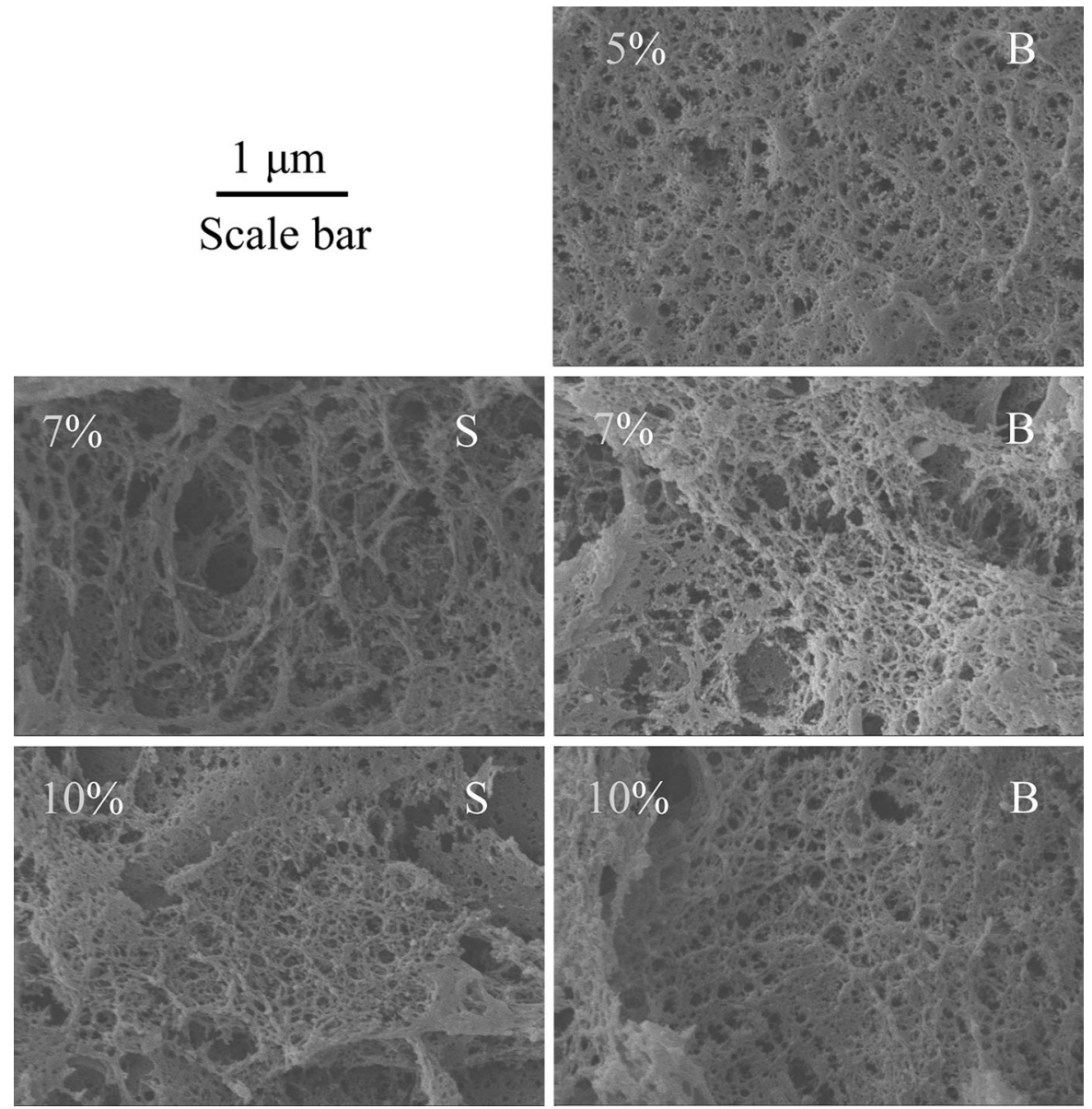

Fig. 7 SEM pictures of the bagasse aerogels at high magnification. $B$ and $S$ refer to bottom side (the face contacted by mold) and upside surface of the aerogels, respectively

\section{Chemical composition of the aerogels}

The FTIR spectra of bagasse, bagasse cellulose and the bagasse aerogel are shown in Fig. 8. The band at $897 \mathrm{~cm}^{-1}$ is assigned to the $\beta$-glucosidic linkages from cellulose. The bands at 1605, 1515 and $834 \mathrm{~cm}^{-1}$ correspond to the ring breathing with $\mathrm{C}-\mathrm{O}$ stretching, aromatic skeletal vibrations and aromatic $\mathrm{C}-\mathrm{H}$ out-ofplan vibration in lignin (Nuopponen et al. 2005). The absorbances at 1735,1378 and $1248 \mathrm{~cm}^{-1}$ are assigned to the $\mathrm{C}=\mathrm{O}$ stretching, $\mathrm{CH}_{3}$ bending and $\mathrm{C}-$ $\mathrm{O}$ antisymmetric stretching of acetyl group in hemicelluloses, respectively. The presence of these bands indicated the successful immobilization of lignin and hemicelluloses into the network of the aerogels. Band height ratios (Fig. 9) were calculated from FTIR to

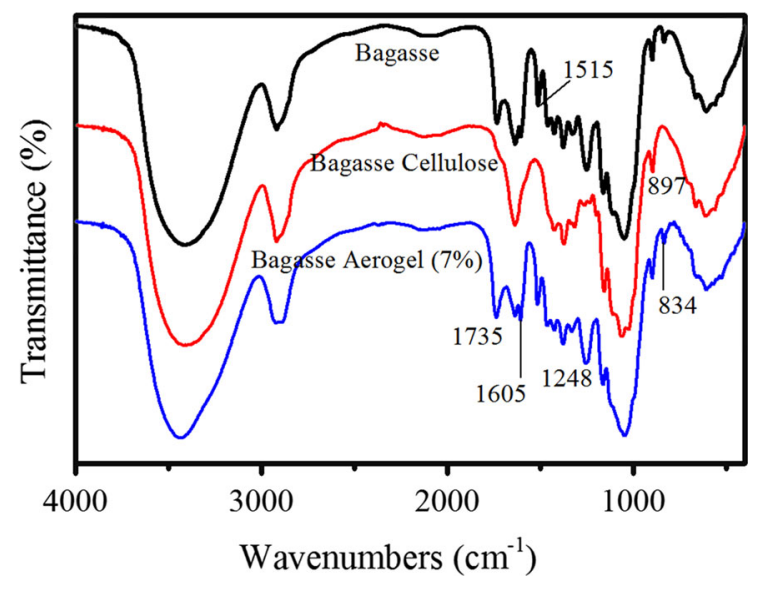

Fig. 8 FTIR spectra of bagasse, bagasse cellulose and the bagasse aerogels 


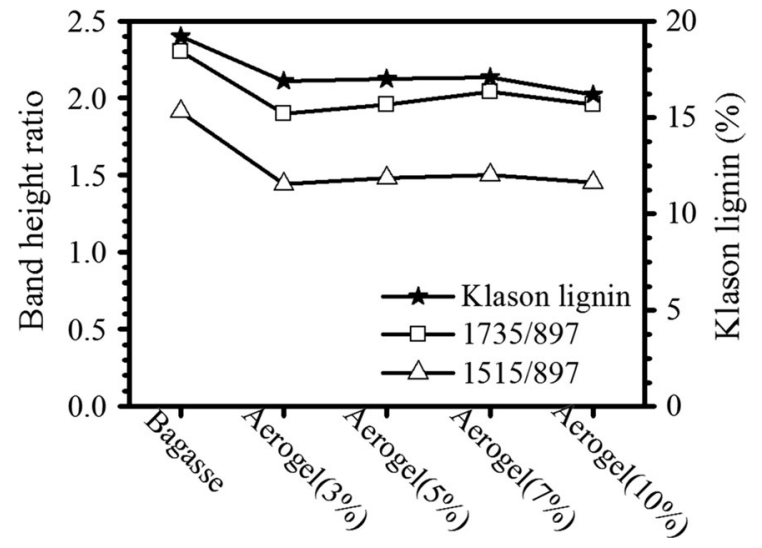

Fig. 9 Quantitative analysis of band height ratios and Klason lignin

determine the quantitative information on the relative content of lignin (1515/893) and hemicelluloses (1735/893). The lignin and hemicellulose content of the aerogels was independent on the bagasse concentration, and lower than that of bagasse. The Klason lignin content of the aerogels and bagasse was also determined. The results from Klason lignin analysis were in agreement with those of band height ratios analysis. These results suggested that a small amount of lignin and hemicellulose was washed out in the preparation of the aerogels.

\section{XRD analysis}

XRD curves of native bagasse, cellulose II, ball-milled bagasse and the bagasse aerogels are shown in Fig. 10. The diffraction peaks of native bagasse at $15.8^{\circ}, 22.0^{\circ}$ and $34.7^{\circ}$ are assigned to Miller indices of (110), (200) and (004) of cellulose I pattern (French 2014), respectively. There were no such diffraction signals at $15.8^{\circ}$ and $34.7^{\circ}$ for the ball-milled bagasse, and a trace of cellulose II diffraction signals were found in the ball-milled bagasse sample. The changes of the crystalline structure of bagasse upon ball-milled treatment were accounted for the successful dissolution of bagasse in $\mathrm{DMSO} / \mathrm{LiCl}$. The dissolution of ball-milled bagasse as well as the subsequent treatment with the aerogel forming processes resulted in the cellulose II pattern of bagasse aerogels. Upon increasing the bagasse concentration from 5 to $7 \%$, and further to $10 \%$, a weak diffraction peak at $34.7^{\circ}$

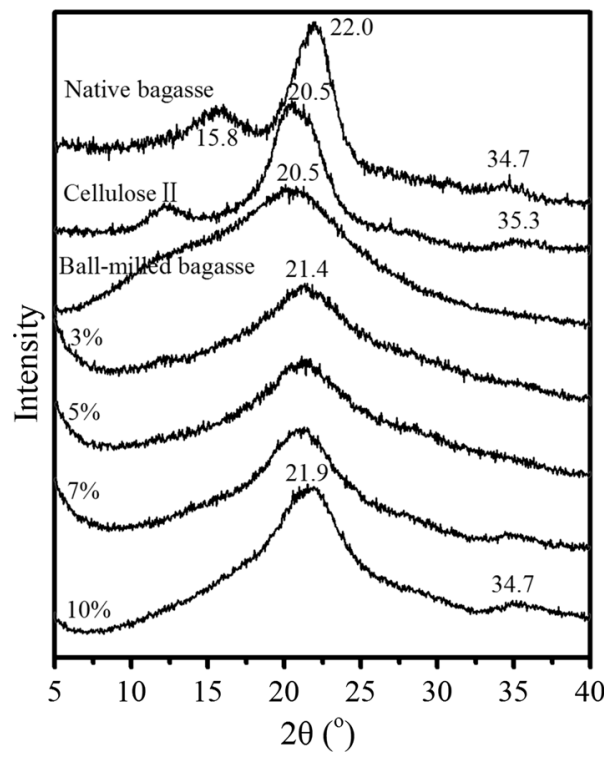

Fig. 10 XRD curves of native bagasse, cellulose II, ball-milled bagasse and the bagasse aerogels

was observed for the aerogels, implying that the changes of the crystalline structure maybe responded to those of the BET surface area and pore volume with the increasing bagasse concentration.

\section{Conclusions}

In summary, highly porous lignocellulosic biomass aerogels were prepared directly from bagasse solutions. The bagasse aerogels displayed sheet-like skeletons, and the highest BET surface areas and pore volumes of the prepared aerogels were $185 \mathrm{~m}^{2} / \mathrm{g}$ and $0.46 \mathrm{~cm}^{3} / \mathrm{g}$, respectively. The bagasse concentration had significant effect on the aerogel preparation. Too low bagasse concentration ( $1 \%$ ) in $\mathrm{DMSO} / \mathrm{LiCl}$ led to the failed preparation of bagasse aerogel, while high concentration $(10 \%)$ resulted in aerogels with noticeably decreased BET surface area and pore volume due to the extensive aggregation of bagasse.

Acknowledgments This work was financially supported by the National Natural Science Foundation of China (31170550 and 31170555), Program for New Century Excellent Talents in University (NCET-11-0154), the Fundamental Research Funds for the Central Universities, and National Program for Support of Top-notch Young Professionals. 
Open Access This article is distributed under the terms of the Creative Commons Attribution 4.0 International License (http:// creativecommons.org/licenses/by/4.0/), which permits unrestricted use, distribution, and reproduction in any medium, provided you give appropriate credit to the original author(s) and the source, provide a link to the Creative Commons license, and indicate if changes were made.

\section{References}

Aaltonen O, Jauhiainen O (2009) The preparation of lignocellulosic aerogels from ionic liquid solutions. Carbohyd Polym 75(1):125-129

Cai J, Kimura S, Wada M, Kuga S, Zhang L (2008) Cellulose aerogels from aqueous alkali hydroxide-urea solution. ChemSusChem 1(1-2):149-154

Chen M, Zhang X, Liu C, Sun R, Lu F (2014) Approach to renewable lignocellulosic biomass film directly from bagasse. ACS Sustain Chem Eng 2(5):1164-1168

French AD (2014) Idealized powder diffraction patterns for cellulose polymorphs. Cellulose 21(2):885-896

Gavillon R, Budtova T (2008) Aerocellulose: new highly porous cellulose prepared from cellulose- $\mathrm{NaOH}$ aqueous solutions. Biomacromolecules 9(1):269-277

Groen JC, Peffer LAA, Perez-Ramirez J (2003) Pore size determination in modified micro- and mesoporous materials. Pitfalls and limitations in gas adsorption data analysis. Microporous Mesoporous Mater 60(1-3):1-17

Guo H, Meador MAB, McCorkle L, Quade DJ, Guo J, Hamilton B, Cakmak M (2012) Tailoring properties of cross-linked polyimide aerogels for better moisture resistance, flexibility, and strength. ACS Appl Mater Interfaces 4(10): 5422-5429

Hao P, Zhao Z, Tian J, Li H, Sang Y, Yu G, Cai H, Liu H, Wong CP, Umar A (2014) Hierarchical porous carbon aerogel derived from bagasse for high performance supercapacitor electrode. Nanoscale 6(20):12120-12129

Heath L, Thielemans W (2010) Cellulose nanowhisker aerogels. Green Chem 12(8):1448-1453

Heath L, Zhu L, Thielemans W (2013) Chitin nanowhisker aerogels. ChemSusChem 6(3):537-544

Hoepfner S, Ratke L, Milow B (2008) Synthesis and characterisation of nanofibrillar cellulose aerogels. Cellulose 15(1):121-129

Hüsing N, Schubert U (1998) Aerogels-airy materials: chemistry, structure, and properties. Angew Chem Int Ed 37(1-2):22-45

Kistler SS (1931) Coherent expanded aerogels and jellies. Nature 127:741
Klemm D, Heublein B, Fink H-P, Bohn A (2005) Cellulose: fascinating biopolymer and sustainable raw material. Angew Chem Int Ed 44(22):3358-3393

Li J, Lu Y, Yang D, Sun Q, Liu Y, Zhao H (2011) Lignocellulose aerogel from wood-ionic liquid solution (1-allyl-3methylimidazolium chloride) under freezing and thawing conditions. Biomacromolecules 12(5):1860-1867

Liebner F, Potthast A, Rosenau T, Haimer E, Wendland M (2008) Cellulose aerogels: Highly porous, ultra-lightweight materials. Holzforschung 62(2):129-135

Lu Y, Sun Q, Yang D, She X, Yao X, Zhu G, Liu Y, Zhao H, Li J (2012) Fabrication of mesoporous lignocellulose aerogels from wood via cyclic liquid nitrogen freezing-thawing in ionic liquid solution. J Mater Chem 22(27):13548-13557

Nagarajan V, Mohanty AK, Misra M (2013) Sustainable green composites: value addition to agricultural residues and perennial grasses. ACS Sustain Chem Eng 1(3):325-333

Nuopponen M, Vuorinen T, Jämsä S, Viitaniemi P (2005) Thermal modifications in softwood studied by FT-IR and UV resonance raman spectroscopies. J Wood Chem Technol 24(1):13-26

Pierre AC, Pajonk GM (2002) Chemistry of aerogels and their applications. Chem Rev 102(11):4243-4266

Sehaqui H, Zhou Q, Berglund LA (2011) High-porosity aerogels of high specific surface area prepared from nanofibrillated cellulose (NFC). Compos Sci Technol 71(13):1593-1599

Sescousse R, Gavillon R, Budtova T (2011) Aerocellulose from cellulose-ionic liquid solutions: preparation, properties and comparison with cellulose- $\mathrm{NaOH}$ and cellulose-NMMO routes. Carbohyd Polym 83(4):1766-1774

Sing KSW (1985) Reporting physisorption data for gas/solid systems with special reference to the determination of surface area and porosity. Pure Appl Chem 57(4):603-619

Sun N, Li W, Stoner B, Jiang X, Lu X, Rogers RD (2011) Composite fibers spun directly from solutions of raw lignocellulosic biomass dissolved in ionic liquids. Green Chem 13(5):1158-1161

Tan C, Fung BM, Newman JK, Vu C (2001) Organic aerogels with very high impact strength. Adv Mater 13(9):644-646

Wang Z, Yokoyama T, H-m Chang, Matsumoto Y (2009) Dissolution of beech and spruce milled woods in $\mathrm{LiCl} / \mathrm{DMSO}$. J Agric Food Chem 57(14):6167-6170

Wang Z, Liu S, Matsumoto Y, Kuga S (2012) Cellulose gel and aerogel from LiCl/DMSO solution. Cellulose 19(2):393399

Wu Z-Y, Li C, Liang H-W, Chen J-F, Yu S-H (2013) Ultralight, flexible, and fire-resistant carbon nanofiber aerogels from bacterial cellulose. Angew Chem Int Ed 52(10):2925-2929

Xie H, Jarvi P, Karesoja M, King A, Kilpelainen I, Argyropoulos DS (2009) Highly compatible wood thermoplastic composites from lignocellulosic material modified in ionic liquids: preparation and thermal properties. J Appl Polym Sci 111(5):2468-2476 